\title{
A DECOMPOSITION PROPERTY OF WEAK $L_{\hat{\imath}}$
}

\author{
by N. T. PECK
}

(Received 14th March 1990)

\begin{abstract}
Let $\left(f_{i}\right)_{i=1}^{n}$ be a set of disjointly supported, positive functions in the Banach envelope of weak $L_{1}$. We prove that each $f_{i}$ can be written as $e_{i}+g_{i}$, where $e_{i}$ and $g_{i}$ are disjointly supported and satisfy these additional properties: the $e_{i}$ 's are isometrically the $l_{\text {co }}^{n}$ basis in the envelope norm; the envelope norm of a linear combination of the $g_{i}$ 's is equal to the envelope norm of the corresponding combination of the $f_{i}$ 's.
\end{abstract}

1980 Mathematics subject classification (1985 Revision). 46B30, 46E30.

In Lotz-Peck-Talagrand $[2,3]$, it was proved that the Banach envelope of Weak $L_{1}(0,1)$ (which we denote by Weak $L_{i}$ ) is universal as a Banach lattice for separable Banach lattices. These two papers also show that a number of non-separable Banach lattices are lattice-isometric to sublattices of Weak $L_{\hat{1}}$.

In this note we show a connection between $l_{\infty}^{n}$ and the structure of Weak $L_{i}$ as a Banach lattice. Before we state the result, recall that the envelope semi-norm on Weak $L_{1}$, which we denote by $\|\cdot\|_{w}$, is given by

$$
\|f\|_{w}=\varlimsup_{\substack{q / p \rightarrow \infty \\ 0<p<q}}\left(\int_{p<|f|<q}|f|\right) / \ln (q / p)
$$

for $f$ in Weak $L_{1}$; see Cwickel-Fefferman [1]. And then Weak $L_{1}$ is the completion of (Weak $L_{1},\|\cdot\|_{w}$ ) modulo the elements $x$ of the completion with $\|x\|_{w}=0$. Under the usual quotient order, Weak $L_{\hat{1}}$ is a Banach lattice.

Our result now is:

Theorem. For $1 \leqq i \leqq n$, let $f_{i}>0$ be elements of Weak $L_{1}$ such that the $f_{i}$ have pairwise disjoint supports, and such that $\left\|f_{i}\right\|_{\mathrm{w}}=1$ for each $i$. Then there exist functions $e_{i}$, $0<e_{i} \leqq f_{i}$ so that

(i) $\left\|\sum_{i=1}^{n} a_{i} e_{i}\right\|_{w}=\sup \left|a_{i}\right|$ for all $\left(a_{i}\right)_{i=1}^{n}$;

(ii) if we set $g_{i}=f_{i}-e_{i}$, then

$$
\left\|\sum_{i=1}^{n} a_{i} f_{i}\right\|_{w}=\left\|\sum_{i=1}^{n} a_{i} g_{i}\right\|_{w} \text { for all }\left(a_{i}\right)_{i=1}^{n}
$$


Proof. First, some notation: for a positive function $f$ in Weak $L_{1}$ and $0<p<q$, denote by $f_{p}^{q}$ the product of $f$ by the indicator function of the set $\{p<f<q\}$. Also, $\|\cdot\|_{w}$ is a lattice semi-norm so in calculating the norm of a linear combination of disjointly supported functions, we can assume all the coefficients are non-negative.

Turning now to the proof, whose arguments are very similar to those of [2] and [3], we first show how to achieve condition (i) of the conclusion. We then show how to achieve condition (ii) by strengthening the arguments of the first part of the proof.

To start, choose a positive summable decreasing sequence $\left(\varepsilon_{m}\right)$ and positive sequence $k_{m} \rightarrow \infty$ so that

$$
q / p>k_{m} \text { implies } \int\left(f_{i}\right)_{p}^{q} / \ln (q / p)<1+\varepsilon_{m} \text { for each } i
$$

Now choose, by induction, positive sequences $\left(p_{i, m}\right),\left(q_{i, m}\right), 1 \leqq i \leqq n, m=1,2, \ldots$ satisfying

$$
\begin{gathered}
q_{i, m} / p_{i, m}>k_{m} ; \\
1-\varepsilon_{m}<\int\left(f_{i}\right)_{p i, m}^{q_{i, m}} / \ln \left(q_{i, m} / p_{i, m}\right) ; \\
0<p_{i, m}<q_{i, m}<p_{i+1, m} \ldots, \quad 1 \leqq i \leqq n-1 ; \\
q_{n, m}<p_{1, m+1} ; p_{i, m} \rightarrow \infty \quad \text { for each } i ; \\
\ln \left(q_{i, m} / p_{i, m}\right) / \ln \left(p_{i+1, m} / q_{i, m}\right)<\varepsilon_{m}, \quad 1 \leqq i \leqq n-1 ; \\
\ln \left(q_{n, m} / p_{n, m}\right) / \ln \left(p_{1, m+1} / q_{n, m}\right)<\varepsilon_{m} .
\end{gathered}
$$

Note that it is possible to choose $p_{i, m}$ arbitrarily large since $\|f\|_{w}=0$ for every bounded $f$ in Weak $L_{1} ;(1)$ and (3) are possible simply because $\left\|f_{i}\right\|_{w}=1$ for each $i$.

Now define

$$
e_{i}=\sum_{m=1}^{\infty}\left(f_{i}\right)_{p_{i, m}, m}^{q_{i, m}} \leqq f_{i}
$$

From (1)-(3) it is clear that $\left\|e_{i}\right\|_{w}=1$ for each $i$. To prove that $\left\|\sum_{i=1}^{n} a_{i} e_{i}\right\|_{w}=\sup \left|a_{i}\right|$, it is enough to prove that

$$
\left\|\sum_{i=1}^{n} e_{i}\right\|_{w}=1
$$

since $\|\cdot\|_{w}$ is a lattice semi-norm.

Suppose now that $0<p<q$, with

$$
0<q_{i-1, j} \leqq p \leqq p_{i, j}<q_{i, j}<p_{i+1, j} \ldots<p_{k, m}<q_{k, m} \leqq q<p_{k+1, m} .
$$


Then

$$
\begin{aligned}
& \int\left(\sum_{l=1}^{m} e_{l}\right)_{p}^{q} / \ln (q / p) \leqq\left[\int\left(f_{i}\right)_{p_{i, j}}^{q_{i, j}} / \ln \left(q_{i, j} / p_{i, j}\right)\right]\left[\ln \left(q_{i, j} / p_{i, j}\right) \ln (q / p)\right] \ldots \\
& +\left[\int\left(f_{k}\right)_{p_{k, m}}^{q_{k, m}} / \ln \left(q_{k, m} / p_{k, m}\right)\right]\left[\ln \left(q_{k, m} / p_{k, m}\right) / \ln (q / p)\right] \\
& <1+\varepsilon_{j}(\text { from (4)) } \\
& \rightarrow 1 \text { as } q / p \rightarrow \infty, p \rightarrow \infty \text {. }
\end{aligned}
$$

If, say,

$$
p_{i, j}<p<q_{i, j} \leqq q<p_{i+1, j}
$$

or

$$
q_{i, j} \leqq p \leqq p_{i+1, j}<q<q_{i+1, j}
$$

it is easy to see that

$$
\int\left(\sum_{l=1}^{n} e_{l}\right)_{p}^{q} / \ln (q / p) \leqq 1+\varepsilon_{j} \quad \text { if } \quad q / p>k_{j}
$$

If

$$
p_{i, j}<p<q_{i, j}<p_{i+1, j} \ldots<p_{k, m}<q<q_{k, m},
$$

then

$$
\begin{aligned}
& \int\left(\sum_{l=1}^{n} e_{l}\right)_{p}^{q} / \ln (q / p) \leqq\left[\int\left(f_{i}\right)_{p_{i, j}}^{q_{i, j}} / \ln \left(q_{i, j} / p_{i, j}\right)\right]\left[\ln \left(q_{i, j} / p_{i, j}\right) / \ln (q / p)\right]+\ldots \\
& +\int\left(f_{k}\right)_{p}^{q} / \ln (q / p) \quad \text { (for the last term) } \\
& \leqq n \sum_{l=j}^{m}\left(1+\varepsilon_{l}\right) \varepsilon_{l}+1+\varepsilon_{m}(\text { by }(5)) \\
& \rightarrow 1 \text { as } j \rightarrow \infty, q / p \rightarrow \infty,
\end{aligned}
$$

using the fact that $\left(\varepsilon_{l}\right)$ is summable.

There are two more cases, depending on the positions of $p$ and $q$ relative to $\left(p_{i, j}\right)$ and 
$\left(q_{i, j}\right)$. Their treatment is similar to that of the cases already discussed, and we leave these cases to the reader.

This shows part (i) of the conclusion.

To achieve part (ii), we have to choose $p_{i, m}, q_{i, m}$ so that the intervals $\left(p_{i, m}, q_{i, m}\right)$ occur sparsely enough on the line. The way to make this precise is to redo one of the arguments in [3]; we start by letting $\left(a_{i, m}\right)$ be an indexing of all the $n$-tuples of positive rational numbers, $m=1,2, \ldots$, with each such $n$-tuple occurring infinitely often in the indexing.

For each $m$, there are $0<r<s$ with $s / r>k_{m}$ and such that

$$
\int\left(\sum_{i=1}^{n} a_{i, m} f_{i}\right)_{r}^{s} / \ln (s / r) \geqq\left(1-\varepsilon_{m}\right)\left\|\sum_{i=1}^{n} a_{i, m} f_{i}\right\|_{w},
$$

simply from the definition of $\|\cdot\|_{w}$. We may choose $r$ as large as we please. Now,

$$
\left(\sum_{i=1}^{n} a_{i, m} f_{i}\right)_{r}^{s}=\sum_{i=1}^{n} a_{i, m}\left(f_{i}\right)_{r / a_{i, m}}^{s / a_{i, m}}
$$

So if we have chosen $p_{i, m}, q_{i, m}$ at the $m$ 'th step in the construction for part (i), we choose $0<r_{m}<s_{m}$ so that

(a) $s_{m} / r_{m}>k_{m}$;

(b) inequality (7) above is satisfied with $r$ and $s$ replaced by $r_{m}$ and $s_{m}$;

(c) $r_{m} / a_{i, m}>q_{i, m}$.

Now return to the construction for part (i), making sure that $p_{i, m+1}$ is chosen so that $s_{m} / a_{i, m}<p_{i, m+1}$. Thus the intervals $\left(p_{i, m}, q_{i, m}\right),\left(r_{m} / a_{i, m}, s_{m} / a_{i, m}\right)$, and $\left(p_{i, m+1}, q_{i, m+1}\right)$ are pairwise disjoint. This dovetailing construction leaves undisturbed part (i) of the conclusion of the theorem. With $g_{i}=f_{i}-e_{i},(8)$ also yields that

$$
\int\left(\sum_{i=1}^{n} a_{i, m} g_{i}\right)_{r_{m}}^{s_{m}} / \ln \left(s_{m} / r_{m}\right) \geqq\left(1-\varepsilon_{m}\right)\left\|\sum_{i=1}^{n} a_{i, m} f_{i}\right\|_{w}
$$

Every $n$-tuple of positive rationals occurs infinitely often in the indexing, so (9) implies that if $\left(a_{i}\right)_{i=1}^{n}$ is any $n$-tuple of positive rationals, then

$$
\left\|\sum_{i=1}^{n} a_{i} g_{i}\right\|_{w} \geqq\left\|\sum_{i=1}^{n} a_{i} f_{i}\right\|_{w}
$$

Finally, by approximating $n$-tuples of non-negative reals by $n$-tuples of positive rationals, we see that $(10)$ is true if $\left(a_{i}\right)_{i=1}^{n}$ is any $n$-tuple of non-negative real numbers. Since $0 \leqq g_{i} \leqq f_{i}$ and the $f_{i}$ are disjointly supported, the inequality 


$$
\left\|\sum_{i=1}^{n} a_{i} g_{i}\right\|_{w} \leqq \sum_{i=1}^{n} a_{i} f_{i} \|_{w}
$$

is automatic for any $n$-tuple $\left(a_{i}\right)_{i=1}^{n}$ of real numbers. The proof of the theorem is complete.

Remark. Let $X$ be an $n$-dimensional Banach lattice which is spanned by $n$ positive disjoint elements $x_{i}$, with $\left\|x_{i}\right\|=1$ for each $i$.

Let $Y$ be the Banach lattice $X \oplus l_{\infty}^{n}$, with norm given by

$$
\|(x, y)\|_{Y}=\sup \left(\|x\|_{X},\|y\|_{i_{\infty}}\right)
$$

Let $\left(e_{i}\right), 1 \leqq i \leqq n$, be the usual basis for $l_{\infty}^{n}$, and for $1 \leqq i \leqq n$ set $z_{i}=\left(x_{i}, 0\right)+\left(0, e_{i}\right)$, $w_{i}=\left(x_{i}, 0\right)$. It is easy to check that

$$
\left\|\sum_{i=1}^{n} \alpha_{i} z_{i}\right\|_{Y}=\left\|\sum_{i=1}^{n} \alpha_{i} w_{i}\right\|_{Y}=\left\|\sum_{i=1}^{n} \alpha_{i} x_{i}\right\|_{X}
$$

for all $\alpha_{1}, \ldots, \alpha_{n}$. And it is obvious from the construction that

$$
\left\|\sum_{i=1}^{n} \alpha_{i}\left(z_{i}-w_{i}\right)\right\|_{Y}=\sup \left|\alpha_{i}\right| .
$$

In $Y$, let $W$ be the span of the $w_{i}$ and $Z$ be the span of the $z_{i}$. From the above, it is clear that both projections $w+z \rightarrow w$ and $w+z \rightarrow z$ of $Y=W+Z$ on to $W$ and $Z$ have norm at least $\left\|\sum_{i=1}^{n} x_{i}\right\|_{X}$.

Our theorem can be used to give a construction of a $2 n$-dimensional Banach lattice $Y^{\prime}$ with the above properties, but which is isometrically different from the $Y$ above, in general. The construction goes as follows: let $X$ be the given Banach lattice spanned by the positive disjoint elements $x_{i}, 1 \leqq i \leqq n,\left\|x_{i}\right\|=1$ for each $i$.

By [2], [3], we may regard $X$ as a sublattice of (weak $L_{1},\|\|_{w}$ ), with the $x_{i}$ corresponding to non-negative, norm-one functions $f_{i}$ with pairwise disjoint supports. On applying the theorem, we have $f_{i}=e_{i}+g_{i}$, with the conclusions of the theorem. Set $Y^{\prime}=\operatorname{span}\left\{e_{i}, f_{j}\right\} 1 \leqq i, j \leqq n$, and define $\left\|\cdot|\||\right.$ on $Y^{\prime}$ by

$$
\left\|\sum\left(\alpha_{i} f_{i}+\beta_{i} g_{i}\right)\right\|=\left\|\sum_{i=1}^{n}\left(\alpha_{i} f_{i}-\beta_{i} g_{i}\right)\right\|_{w}
$$

Then with $f_{i}$ corresponding to $z_{i}$ and $g_{i}$ corresponding to $w_{i}$, properties (11) and (12) are immediate for $\left(f_{i}\right),\left(g_{i}\right)$. 
Suppose now that $X$ is $l_{1}^{n}$. The canonical way of embedding $X$ into (weak $L_{1},\|\|_{w}$ ) is to let each basis vector $x_{i}$ correspond to a function $f_{i}$ so that for each $i$, the decreasing rearrangement of $f_{i}$ at $t$ is $1 / t$, for sufficiently small $t$. If $n \geqq 2$, then for $m$ sufficiently large,

$$
\begin{aligned}
\int\left(e_{1}+g_{2}\right)_{p_{1, m}}^{q_{1, m}} & =\int\left(e_{1}+f_{2}-e_{2}\right)_{p_{1, m}}^{q_{1, m}} \\
& =\int\left(e_{1}+f_{2}\right)_{p_{1, m}}^{q_{1, m}} \\
& =2 \ln \left(q_{1, m} / p_{1, m}\right),
\end{aligned}
$$

since $e_{2} \chi_{p_{1, m}}<e_{2}<q_{1, m}=0$. Thus $\left\|e_{1}+g_{2}\right\|_{w}=2$, showing that there is no isometry of $Y^{\prime}$ onto $Y$ which takes $f_{i}$ to $z_{i}$ and $g_{i}$ to $w_{i}$.

\section{REFERENCES}

1. M. Cwikel and C. Fefferman, Maximal seminorms on weak $L_{1}$, Studia Math. 69 (1980), 149-154.

2. H. P. Lotz and N. T. Peck, On Banach sublattices of weak $L_{1}$, Proc. Amer. Math. Soc., to appear.

3. N. T. Peck and M. Talagrand, Banach sublattices of Weak $L_{1}$, Israel J. Math. 59 (1987), 257-271.

UNIVERSITY OF ILLINOIS

Department of Mathematics

1409 West Green Street

Urbana, IlLinoIs 61801, USA 